\title{
Utilization of Soft Computing Techniques for Improving the Traditional Technical Analysis in the Financial Markets
}

\author{
Norio Baba, Osaka Kyoiku University \\ Tomoko Kawachi, Osaka Kyoiku University \\ Toshinori Nomura, Osaka Kyoiku University \\ E-mail: baba@cc.osaka-kyoiku.ac.jp \\ Tel\&Fax: 0729-78-3670
}

\begin{abstract}
During the last several decades, the soft computing techniques such as NNs \& GAs have been studied quite extensively by many researchers and have been applied to various real world problems. They have been successfully utilized for constructing a large number of intelligent systems. One of the most remarkable application areas may be the financial market. Nowadays, comparatively large numbers of researchers show keen interest in the application of soft computing techniques to the financial market.

In this paper, we shall propose a DSS for dealing stocks which improves the traditional technical analysis by utilizing soft computing techniques. In the proposed DSS, soft computing techniques are utilized in order to detect "Golden Cross" and "Dead Cross" several weeks before it occurs. We shall also give several computer simulation results which confirm the effectiveness of the proposed DSS.
\end{abstract}

\section{Introduction}

One fundamental aspect of neural networks (NNs) is their capability to build complex nonlinear relationships between input variables and output variables directly from the training data. This characteristic feature of $\mathrm{NNs}$ is especially suited to various applications. In recent years, large numbers of researchers have had strong interest in the application of NNs to the financial market [1]-[10]. There have so far been presented various interesting approaches to construct intelligent decision support systems for dealing stocks.

In this paper, we shall propose a new DSS for dealing stocks which improves the traditional technical analysis by utilizing NNs. In the proposed system, NNs are utilized in order to predict the "Golden Cross ("Dead Cross") several weeks before it occurs.
The structure of this paper is as follows: In Section 2, we briefly touch upon the traditional technical analysis which utilizes the measure "long term moving average" versus "short term moving average" for finding the current tendency of the stock market. In Section 3, we propose a modified DSS for dealing stocks which utilizes NNs in order to predict the "Golden Cross" ("Dead Cross") several weeks before it actually occurs. Section 4 gives several computer simulation studies which confirm the effectiveness of the proposed DSS. Finally, the paper concludes with a brief summary.

\section{Traditional Technical Analysis Utilizing the Measure "Long Term Moving Average Versus Short Term Moving Average"}

In order to detect the current tendency of the stock market, many stock traders have so far used the traditional technical analysis which utilizes the measure "long term moving average (LTMA) versus short term moving average (STMA)". Figure 1 and Figure 2 illustrate the golden cross and the dead cross of the two moving averages, respectively. As is well known among many stock traders, "Golden Cross" ("Dead Cross") is one of the most familiar indicators that predict that the stock price will become higher (lower). Therefore, many people have often utilized these measures for dealing stocks.

The prototype DSS utilizing the traditional technical analysis can be described as follows:

1) If the sign of (the LTMA (such as 13 weeks moving average) - the STMA (such as 5 weeks moving average) ) changes from positive to negative, then buy stocks.

2) If the sign of (the LTMA (such as 13 weeks moving average) - the STMA (such as 5 weeks moving average) ) changes from negative to positive, then sell stocks. 


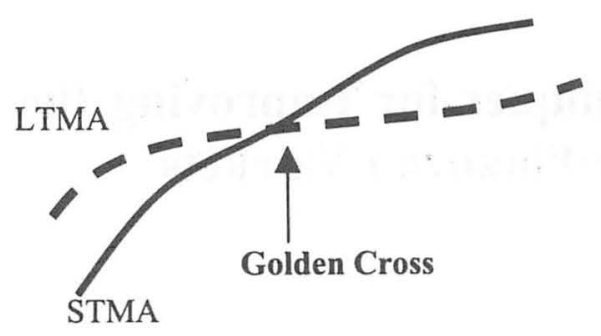

Fig. 1. Golden Cross of the two moving averages

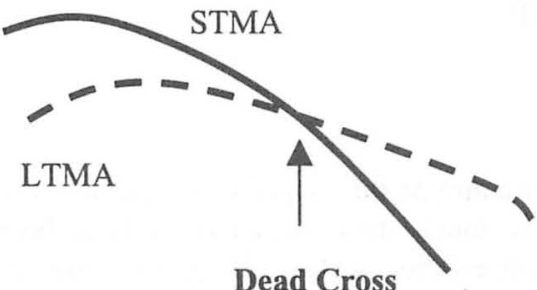

Fig.2. Dead Cross of the two moving averages

\section{A New Decision Support System for Dealing Stocks Which Improves the Traditional Technical Analysis}

The traditional technical analysis utilizing the measure LTMA versus STMA has been widely recognized as one of the most reliable techniques for dealing stocks. However, we consider that the traditional technical analysis could further be improved by the use of NNs. The prototype DSS utilizing the traditional technical analysis executes dealings after golden cross or dead cross has been found. However, such a way of dealings often loses an important chance to execute dealings in a reasonable price in the stock market. If one could deal stocks several weeks before real crossing occurs, one could save considerable amount of money.

In this section, we shall propose a decision support system (DSS) for dealing stocks which utilizes the information (concerning the possibility of the "Golden Cross" ("Dead Cross")) having been obtained by the use of NNs effectively.

\subsection{Prediction of the crossings between the STMA and the LTMA by NNs}

\subsubsection{Choosing the input variables into the neural} network model

In order to construct a DSS using NNs which makes appropriate prediction of the crossings between the STMA and the LTMA, one has to choose input variables carefully which may give significant effect upon the changes of the two moving averages of the stock price. We have utilized the sensitivity analysis for this objective.[4],[5]

\subsubsection{Teacher Signals}

The proposed decision support system (DSS) has been constructed in order to predict crossing (Golden Cross or Dead Cross) several weeks before it occurs. In order to let the DSS have such an intelligent property, we have prepared the teacher signals for neural network training. The following figure (Fig.3) illustrates how the teacher signals (near to the week when the Golden Cross has been observed) have been constructed. (We shall omit explanations relating to the teacher signals corresponding to the Dead Cross.)

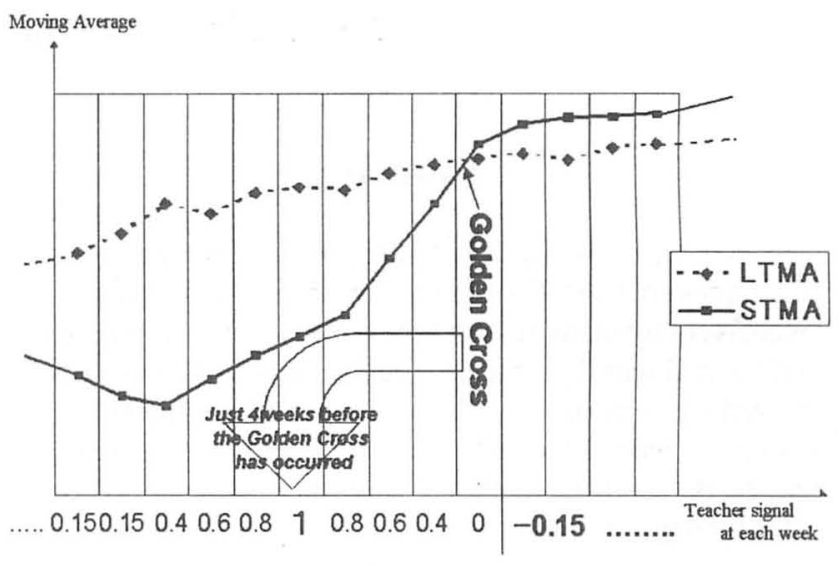

Fig.3 Teacher signals near to the week when the Golden Cross has been observed.

\subsubsection{Neural Network Training}

Neural network training has been carried out by using MATLAB Toolbox in order to make a prediction of the "Golden Cross" and "Dead Cross" several weeks before they occur.

\subsection{Decision Support System for Dealing Stocks}

By utilizing outputs from the trained neural network, one can make a prediction of the crossings "Golden Cross" and "Dead Cross" several weeks before they occur. This means that one can use the information concerning outputs obtained from the neural network (NN) effectively in order to carry out dealing stocks.

Below, let us propose a new decision support system (DSS; Fig.4) which utilizes this information effectively for dealing stocks: 


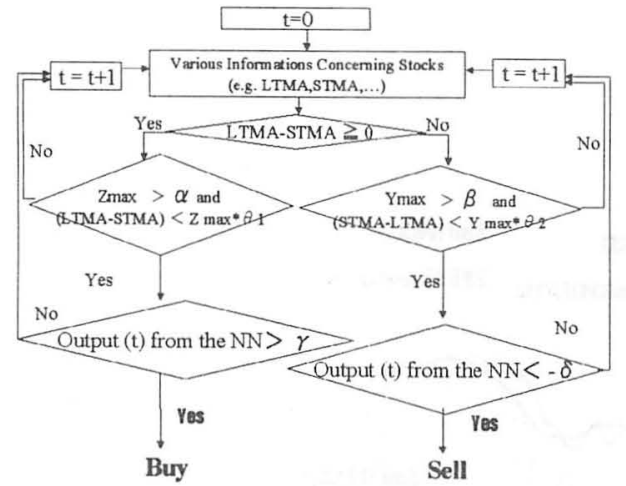

Fig.4 Decision support system for dealing stocks which utilizes NNs

Remark 3.1 Zmax (Ymax) denotes the maximum difference between LTMA and STMA(STMA and LTMA) having been observed after the recent Dead Cross (Golden Cross).

Remark 3.2 The condition $\mathrm{Zmax}>\alpha(\mathrm{Ymax}>\beta)$ in the Figure 4 is used in order to guarantee the maximum gap having been observed after the recent Dead Cross (after the recent Golden Cross) between LTMA and STMA to be large enough. Further, the condition LTMA - STMA < $Z \max * \theta_{1}\left(\right.$ STMA - LTMA $\left.<Y \max * \theta_{2}\right)$ is introduced in order to guarantee the difference between LTMA and STMA to have been shortened considerably.

Remark 3.3 If the two conditions in the Remark 3.2 are satisfied and the output from the trained neural network surpasses the threshold $\gamma$, then the proposed DSS makes the decision "Buy". If the two conditions in the parentheses in the Remark 3.2 are satisfied and the output from the trained neural network falls below the threshold - $\delta$, then the proposed DSS makes the decision "Sell".

\section{Computer Simulations}

We have carried out computer simulations for dealings in the Nikkei-225 from 1997 to 1999. Table 1 shows the changes of the initial money ( 10 billion yen) by the dealings in the Nikkei-225 utilizing the proposed DSS, the traditional technical analysis, and the Buy-and-Hold method. These simulation results demonstrate that the proposed DSS outperforms the traditional technical analysis and the Buy-and-Hold method considerably. (Although the traditional DSS and Buy-and-Hold method have not been able to produce return, the proposed DSS has succeeded in yielding around $15 \%$ average return each year.) In order to demonstrate how the proposed DSS has behaved intelligently in dealing Nikkei-225, we shall give the computer simulation results concerning the dealings by the proposed DSS, the traditional approach, and Buy-and-Hold Method during 1997. (Fig.5)
Table 1. Computer Simulation Results (Nikkei 225; From 1997 to 1999)

\begin{tabular}{|c|c|c|c|}
\hline & $\begin{array}{c}\text { Proposed } \\
\text { DSS }\end{array}$ & $\begin{array}{c}\text { Traditional } \\
\text { DSS }\end{array}$ & Buy\&Hold \\
\hline $\begin{array}{c}\text { Initial } \\
\text { Amount of } \\
\text { Money }\end{array}$ & 10.000 & 10.000 & 10.000 \\
\hline $\begin{array}{c}\text { Final } \\
\text { Amount of } \\
\text { Money }\end{array}$ & 14.470 & 9.726 & 9.584 \\
\hline Returns & +4.470 & -0.270 & -0.415 \\
\hline
\end{tabular}

(10 billion yen)

Remark 4.1 In the above computer simulations, we have taken the charge for dealing into account by subtracting $\{0.001 *$ (total money used for dealing $)+250,000\}$ yen from the total fund for dealing.

Remark 4.2 In the above computer simulations, the following parameter values have been used:

$$
\begin{aligned}
& \alpha=300, \quad \beta=300, \quad \gamma=0.65, \quad \delta=0.65 \\
& \theta_{1}=0.85 \quad \theta_{2}=0.85
\end{aligned}
$$

Remark 4.3 In the above computer simulations, we have not taken "selling on credit" into account. We have just followed the rule: "Buy first, and then sell" We have also followed the following rules:

1) Buy stocks by using all of the money on hand when the DSS has made the decision "buy".

2) Sell all of the stocks on hand when the DSS has made the decision "sell".

\section{Concluding Remarks}

A decision support system (DSS) for dealing Nikkei-225 which improves the traditional technical analysis is developed in this paper. NNs are utilized in order to predict the "Golden Cross" ("Dead Cross") several weeks before it actually occurs. Computer simulations which have been carried out confirm the effectiveness of the proposed DSS using NNs. However, these simulations have been done during a rather short range of years. In order to execute full confirmation concerning the developed DSS, we need to carry out huge numbers of simulations through a rather long range of years.

The authors believe that the idea proposed in this paper could be successfully applied for dealing in the various indices (such as TOPIX) and the listed stocks in this world. Future research effort should be directed for checking this possibility.

Further research is also needed in order to investigate whether the proposed approach can be appropriately applied 
to the various types of the artificial markets and the experimental markets.

\section{Acknowledgements}

The authors would like to thank the partial financial support of the Foundation for Fusion of Science \& Technology (FOST). They also would like to express their thank to QUICK Corporation for their kind support in giving them various financial data.

\section{References}

\section{References}

[1] A.P. Refenes, Editor, Neural Networks in the Capital Markets, Wiley, 1995.

[2] N.Baba and M.Kozaki, "An intelligent forecasting system of stock price using neural network", Proceedings of IJCNN, 1, pp.371-377, 1992.

[3] N.Baba et al. "A hybrid algorithm for finding the global minimum of error function of neural networks and its applications", Neural Networks, Vol.7, pp.1253-1265, 1994.

[4] H.L.Viktor et al, "Reduction of symbolic rules from artificial neural network, using sensitivity analysis", IEEE International Symposium on Circuits and Systems, 1995. [5] J.M.Zurada et al, " Sensitivity analysis for minimization of joint data dimension for feedforward neural network", IEEE International Symposium on Circuits and Systems, 1994.

[6] N.Baba, "Construction of the decision support system for dealing stocks which utilizes neural networks", MTEC Journal, Vol.1, pp.3-41, 1998.

[7] N.Baba and H.Suto, "Utilization of artificial neural networks and TD-Learning method for constructing intelligent bdecision support systems", European Journal of Operational Research, Vol.122, pp.501-508, 2000

[8] N. Baba et al, "Utilization of soft computing techniques for constructing reliable decision support systems for dealing stocks, Proceedings of IJCNN, Hawaii, pp.2150-2155, 2002.

[9] N.Baba and Y.Wang, "Utilization of NNs \& GAs for the design of a reliable decision support system for dealing stocks, Proc. the JAFEE Conference, pp.379-391, 2003.

[10] N.Baba and T.Kawachi, "A new trial for improving the traditional technical analysis in the stock markets", proceedings of the 8th KES conference, New Zealand pp.434-440, September, 2004.

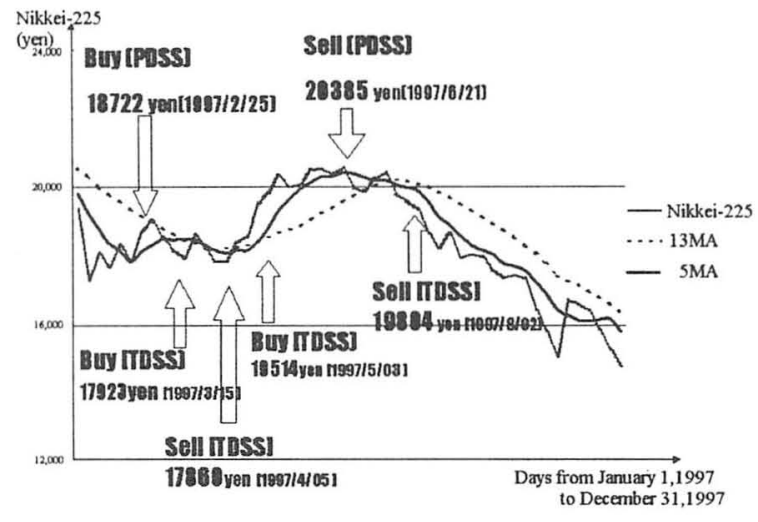

Fig. 5 Dealings by the proposed DSS(PDSS) and the traditional DSS(TDSS). (Year:1997) 